\title{
HISTORIA DEL EJERCICIO PROFESIONAL DE ENFERMERAS HOSPITALARIAS DEL SUR DE CHILE (1940-1980)
}

\author{
HOSPITAL NURSING PRACTICE HISTORY, \\ SOUTH OF CHILE (1940-1980)
}

\author{
Pamela Paredes M. \\ EDITH Rivas R.*
}

\begin{abstract}
RESUMEN
En Chile el ejercicio profesional histórico de las enfermeras ha sido poco documentado. Objetivo: Conocer el ejercicio profesional de enfermeras hospitalarias, años 1940-1980, para develar la identidad profesional y memoria histórica. Método: Estudio histórico, historia oral a través de entrevista semiestructurada, incluyó relatos de vida. Se eligió el contexto de los años 1940-1980, por contar con referentes vivenciales de la época. Los criterios de validez considerados: subjetividad, memoria y particularidad de la fuente. Resultados: Contexto personal, presencia de influencias de los valores de la formación, obediencia a las estructuras de poder, sacrificio personal y sumisión. Contexto laboral, caracterizado por una situación de trabajo extremo y marcada carencia de enfermeras derivado de la insuficiencia de escuelas formadoras en el país. En el rol asistencial se observa déficit en el desarrollo profesional, conformidad, cooperación ciega y carencia de análisis, debido a la gran demanda de atención, por epidemias de diarreas, problemas respiratorios y accidentes. Relevancia en realización de procedimientos: arsenalería, anestesia, asistencia directa en cirugías y administración de quimioterapia. El rol administrativo estuvo caracterizado por: habilitación y organización de servicios, se devela una enfermería estructurada, centradas en la solución de problemas domésticos y en el manejo de recursos humanos, el rol educador: formación formal de auxiliares. Conclusión: La práctica y el saber en el campo de salud están ligados a la transformación histórica, recibiendo en el ejercicio profesional una fuerte influencia de los contextos sociales, políticos y económicos que le acompañan.
\end{abstract}

Palabras clave: Enfermería, práctica profesional, historia de la enfermería.

\begin{abstract}
In Chile the historical practice of nurses has been scarcely documented. Objective: To determine the professional practice of registered nurses, years 1940-1980, to reveal the identity and historical memory. Method: Historical, oral history methodology, data collection through semi-structured interview that included stories of life. We chose the years 1940-1980 as context, because we counted on the experiential referents for that age. As validity criteria, the subjectivity, memory and particularity of the source, were considered. Results: The personal context, influences of training values, obedience to the power structures, self-sacrifice and submission. The work environment is characterized by a situation of extreme work and noticeable lack of nurses resulting from inadequate training schools in the country. In the caring role, any deficit in professional development, compliance, and lack of cooperation and blind analysis, due to the high demand for care, epidemics of diarrhea, respiratory problems and accidents. Of particular importance is carrying of some procedures as giving anesthesia, direct assistance in surgery and chemotherapy. The administrative role was characterized by empowerment and service organization, nursing shows a structured, focused on solving problems of a domestic and human resource management. The educator role consisted on formal training for Nursing Assistants offered by the Training

\footnotetext{
* Enfermera. Docente Escuela de Enfermería. Universidad Santo Tomas. Email: pamelacaro77@gmail.com

${ }^{* *}$ Enfermera. Docente Departamento de Pediatría y Cirugía Infantil. Universidad de la Frontera. Email: edith.rivas@ufrontera.cl
} 
Unit of the Hospital. Conclusion: The overall analysis leads us to conclude that the practice and knowledge in the field of health are linked to the historical transformation of the economic production process, receiving in the professional practice a strong influence of social, political and economic contexts that accompany it.

Key words: Nursing, professional practice, history of nursing.

Fecha recepción: 18/06/12 Fecha aceptación: 30/07/13

\section{INTRODUCCIÓN}

La acepción del Código Deontológico de Enfermería, manifiesta que el ejercicio profesional de las/os enfermeras/os, recibe influencias de las condiciones sociopolíticas y de los acontecimientos históricos (1). Sin embargo, la evolución histórica del ejercicio profesional en las enfermeras representa un área poco documentada en Chile, que invita a descubrir el devenir del mismo, para contribuir al conocimiento histórico e incrementar su saber, constituyéndose en una herramienta capaz de conducir y sustentar la legitimidad, autoridad y autonomía profesional (2).

En esta línea, Verderese manifiesta que el desarrollo de Enfermería está ligado al proceso de industrialización que demanda el desarrollo de la atención médica, a fin de proteger la mano de obra y aumentar su productividad, situación que impulsa un crecimiento vertiginoso de la industria hospitalaria entre 1940 y 1960 (3). Hacia la década de 1950, comienza a esbozarse el desarrollo de la medicina de tipo preventiva, pero no sigue el mismo ritmo de la industria hospitalaria, pasando a ocupar un papel secundario dentro de la práctica de salud. De esta forma, la práctica de la función preventiva la asume el personal sanitarista y está dirigida principalmente a las zonas urbanas y áreas estratégicas (4). En este contexto histórico, Chile recibe la cooperación de organismos internacionales e interamericanos como la Organización Panamericana de la Salud (OPS), Fundación Rockefeller e Instituto de Asuntos Interame- ricanos, entre otros, lo que se intensificó durante la Segunda Guerra Mundial.

Se establecen asimismo los Servicios Cooperativos de Salud, dirigidos a prestar Asistencia Sanitaria en áreas estratégicas, los que desaparecen en el decenio de 1960, integrándose a los Ministerios de Salud. Igualmente se afianza en este período el reconocimiento de la importancia de los Servicios de Enfermería técnicamente competentes, para la prestación de Servicios de Salud, en el área hospitalaria y medicina preventiva. En este contexto fueron factores relevantes para el desarrollo de la enfermería en nuestro país, la posición de la mujer en la sociedad, la apropiación de los conocimientos médicos por la clase médica dominante, que parecen haber influido limitando y demarcando su desarrollo como profesión (3).

En materia de necesidades de recursos, no se conoce el número de enfermeras trabajando en América Latina antes de 1949; pero un estudio realizado por la OPS (5), señala que 5.121 enfermeras trabajaban en los hospitales y 1.124 en Salud Pública. Dicha investigación refiere que a la fecha no se contaba con datos referentes al número y tipo de Escuelas existentes con anterioridad a 1949 (5).

\section{Contexto histórico años 1940-1980}

La creación de nuevos hospitales en el sur de Chile, en el año 1940, causa impacto en la población, siendo reconocidos algunos de estos por su infraestructura de gran modernidad para la época y el desarrollo de las prácticas de enfermería, las cuales ponen énfasis en los 
conocimientos técnicos y se encuentra fuertemente ligada al desarrollo de los hospitales.

Por otra parte, el escenario político de 1950 trae consigo una serie de movimientos sociales que demandan una transformación social (la clase media y obrera empobrecidas realizan demandas al Estado en busca de mejores condiciones de vida y salud). Bajo este argumento entra en vigencia la Ley 10.038, que retomaba el histórico proyecto de Estado Asistencial, ingresado en 1941 y que permanece por más de 10 años en el Congreso para su aprobación (6). Cabe mencionar que desde el año 1942 se encontraban funcionando la Dirección General de Protección de la Infancia y la Adolescencia (PROTINFA), las Unidades Sanitarias, promulgándose la Ley de Asignación Familiar Obrera (1953) y la Asignación Familiar Prenatal (1957) (7).

En la década del 60, Chile sigue siendo un país subdesarrollado con 7 millones de personas, $40 \%$ tenía 15 años de edad o menos (8), las condiciones de vida eran insatisfactorias, con coberturas insuficientes en los servicios básicos y dificultades en el acceso a la educación, vivienda y salud. La tasa de analfabetismo superaba el 25\%, la educación primaria no cubría al 70\% de la población, y de los que la iniciaban sólo un 10\% lograba finalizarla (9). La migración hacia los centros urbanos era muy elevada, lo que provocaba un enorme déficit habitacional, que en ese entonces se estimaba en unas 350.000 viviendas (10); sólo el 40\% de las viviendas contaba con agua potable, y menos de un 20\% estaba conectada con la red del alcantarillado (9). La mortalidad infantil era de 120 por mil nacidos vivos (NV) y la mortalidad del preescolar llegaba a 25 por mil. Cerca del 60\% de las muertes ocurría antes de los 15 años de edad (9), se estima que aproximadamente $60 \%$ de los menores de 6 años, en comunas urbanas de Chile Central, presentaban algún grado de desnutrición (11) lo que contribuía a aumentar considerablemente la mortalidad infantil por enfermedades entéricas y respiratorias. No obstante lo desalentador, en el país se encontraba vigente una legislación cuyo propósito era mejorar la calidad de vida del binomio madre-hijo, Ley No 4.054 del Seguro Obligatorio de Enfermedad e Invalidez, la Ley No 6.174 de Medicina Preventiva y la Ley No 6.236, denominada también Ley de La Madre y El Niño (7). Sin embargo, fue la creación del Servicio Nacional de Salud (SNS) en 1952 la que generó el mayor impulso en el fomento y la protección de la Salud. El SNS agrupó a una serie de instituciones (Beneficencia, Seguro Obrero, Sanidad), asumiendo sus labores y ampliando considerablemente su campo de acción (12). La atención médica del SNS se llevó a cabo en los niveles primarios, secundario y terciario, destacando el funcionamiento de los Centros de Hidratación Ambulatoria, en donde se controlaban los niños con diarreas graves asociadas a desnutrición (12-13).

En el Sur de Chile, un acontecimiento histórico relevante fue el terremoto del año 1960, y por considerarse Zona de Desastre, surge la creación de la Escuela de Enfermería, de la Universidad Austral de Chile, producto también del estudio de recursos humanos y necesidades de Enfermería realizado por Krebs, quien evidencio la urgente necesidad de incrementar la formación de este profesional en el país y firmar el Convenio Chile/ Organización Mundial de la Salud/Fondo de las Naciones Unidas para la Infancia (Chile/ OMS/UNICEF), "Programa de Asistencia al desarrollo de la Enfermería en Chile" (14). La Escuela de Enfermería de la Universidad Austral de Chile nace en 1963, y el Hospital recibe a las primeras alumnas el año 1965, Internas de Enfermería en 1966, año que se titula la primera promoción formada por 28 alumnas. A partir de 1968 surge el Convenio Docente Asistencial de la XI Zona de Salud Valdivia-Osorno, entre el Servicio Nacional de Salud y la Universidad Austral de Chile, con el propósito de contribuir a solucionar la insuficiencia de profesionales enfermeras en la zona. En 1969, en virtud de este convenio se destinaron profesoras a los servicios 
básicos, para asumir responsabilidades asistenciales y docentes (14).

En las décadas siguientes se consolida la formación formal de Auxiliares de Enfermería en la Unidad de Capacitación de los Hospitales Base, conjuntamente emerge el año 1965 la Revista de Enfermería del Colegio de Enfermeras de Chile, considerada como agente facilitador en la información de actividades formativas y de los avances de la Enfermería en Chile. Adicionalmente se recibe la influencia de otras publicaciones internacionales como la revista Nursing, que hacía énfasis en los avances tecnológicos de procedimientos y cuidados de Enfermería.

Posteriormente, se adoptaron medidas tendientes a ampliar la formación de los distintos profesionales del equipo de salud, así entre 1965 y 1970 se crearon siete (7) Escuelas de Enfermería, cinco (5) Escuelas de Matronas y cuatro (4) Escuelas de Nutricionistas (7-15).

Otro hito trascendente fue el golpe militar de 1973, durante el cual se produjeron allanamientos e ingreso de personal militar a los recintos hospitalarios y públicas amenazas, hacia el personal de salud ${ }^{1}$. En 1976 la Enfermería en Chile había tomado un curso de transformación de lo individual e institucionalizado a lo familiar y comunitario, con una población con mayor expectativa de vida (62 y 67 años), con alta incidencia de enfermedades circulatorias, degenerativas e infecciosas. El ejercicio profesional estaba marcado por la hegemonía del poder médico, la falta de interrelación enfermera-paciente, esto último relacionado con el déficit de enfermeras (16). Hacia 1980 se observa mayor crecimiento tecnológico y una fuerte preocupación por las especializaciones médicas; persiste el déficit de enfermeras a nivel nacional mientras que el régimen político de la época disminuye la matrícula de algunas Escuelas de Enfermería del país.

${ }^{1}$ Discurso del ministro Spoerer: Normalización de los Servicios de Salud. Diario El Mercurio 15 de septiembre de 1973.

\section{Ejercicio profesional}

La formación y el ejercicio de Enfermería fue perpetuándose primordialmente en el ámbito hospitalario, desarrollándose un vínculo histórico entre Enfermería como profesión y la ética en su actuar (17). En este sentido, el ejercicio profesional en el área hospitalaria reconoce un pensamiento caracterizado por la rigidez y la obediencia a la jerarquía, probablemente con influencias heredadas de la filosofía Nightingale. Adicionalmente, dicho fenómeno queda afecto a las condiciones socioeconómicas y los acontecimientos históricos que influyen en la profesión (18).

En Chile, en el aspecto académico, se observa mayor comprensión del significado del ejercicio profesional, según lo planteado por Pincheira, "la enfermera profesional es aquella que ha seguido un Programa General de Formación en el Sistema de Educación Superior, lo cual le proporciona amplias y sólidas bases para la práctica efectiva de la Enfermería Profesional y la Formación Superior de Postgrado..." (19). Esta definición ofrece una mirada más amplia del rol profesional, pero al mismo tiempo supone cierto grado de incongruencias entre lo declarado y lo ejecutado en la práctica; de esta manera, un análisis histórico resulta imprescindible, ya que el estudio de la profesión responde a una creación y fundación humana, que no se entienden bien cuándo, cómo y porqué han surgido si no es dentro de la historia como sugiere Gracia. Parafraseando a este autor ¿Por qué la Enfermería es lo que es, en Chile? esto se analiza desde su gestación o no se entiende. Un profesional ha de ser alguien que tenga conciencia de su rol histórico social creado por hombres y sociedades (20). Por otra parte es a partir de 1976 que el Colegio de Enfermeras delimita los lineamientos del ejercicio profesional al definir el rol del profesional de Enfermería en Chile.

De este modo el objetivo de este trabajo es conocer el ejercicio profesional de enfermeras hospitalarias, años 1940-1980, para 
develar la identidad profesional y memoria histórica.

\section{MÉTODO}

En función de la naturaleza y características del problema, se utilizó el paradigma cualitativo (21), respetando al sujeto en su singularidad, reconociendo la importancia del conocimiento, de la experiencia social y de su dimensión política (22). De esta forma se realizó un estudio histórico, con metodología de historia oral, como forma de acercarnos a los sujetos y de llevar al ámbito de lo "público" las voces de los mismos (23).

La recolección de la información se realizó a través de entrevistas semiestructuradas que incluyeron relatos de vida y visiones de mundo. Para la correcta elección de los informantes se utilizó la técnica de bola de nieve, de donde se obtenían datos de nuevos informantes a partir de ellos mismos; éstos debían tipificar procesos históricos, y esta elección dependió de la información buscada y de su utilidad, es decir se seleccionaron personas a partir de sus propias experiencias (24-25).

En el análisis se eligió el contexto de los años 1940-1980, por contar con referentes vivenciales de la época (sujetos que iniciaron su vida laboral en la época y que estuvieron dispuestos a emitir sus relatos). De acuerdo a la selección, las cinco (5) enfermeras cumplieron los criterios de representatividad, por sus condiciones personales, historias y experiencias; criterio de inserción temporal por vivencias de la época y espacio; nivel de participación; por ser partícipe de los acontecimientos. Conjuntamente se consideró la pertinencia temporal al lapso de tiempo que apuntaba este estudio: la edad; interesaba el poder "captar" las diferentes generaciones que participaban del mismo y en elaboración verbal estaba el interés por contar sus historias (26). Igualmente, la capacidad de elaboración verbal de los sujetos.
Para la realización de las entrevistas se registraron las experiencias, ordenados en fases o pausas los acontecimientos, por lo tanto la narrativa fue más allá de la palabra hablada (27). De acuerdo a Hammer y Wildavsky los temas se ordenaron de modo de estimular la memoria (28). Conjuntamente se contó con preguntas orientadoras de diálogo para guiar los relatos (29). Al finalizar, el entrevistado podía solicitar la transcripción de la entrevista integra y tenía derecho a solicitar modificaciones. Siempre la palabra final del entrevistado fue respetada (27). Como criterios de validez fueron considerados: la subjetividad, memoria y particularidad de la fuente, y ésta fue el testimonio dentro del contexto de la entrevista.

Para el análisis se dividieron los años en dos periodos: 1940-1960 y 1970-1980, se consideró relevante diferenciar los períodos ya que en ellos se encuentran hitos históricos (sociales, políticos o económicos) que influyen en el ejercicio profesional de diferente manera y permitió organizar la información recolectada; la transcripción de los registros orales se orientó buscando las comparaciones entre sí, por medio de la categorización de los temas centrales y el agrupamiento por períodos de estudio, lo que aportó claridad (30). De esta manera se obtuvieron códigos y categorías de análisis.

Consideraciones éticas: se consideraron los siete requisitos éticos de Emmanuel'2: valor social, científico o clínico, generando conocimiento respecto de una porción de historia local; validez científica, la que se expresó por el desarrollo de una investigación correctamente diseñada, con rigor metodológico. E igualmente los criterios de Guba (31), en: valor de verdad, aplicabilidad, consistencia y neutralidad, que dan fe de la rigurosidad y veracidad de los resultados, para lo cual se

\footnotetext{
2 Emmanuel E. ¿Qué hace que la investigación clínica sea ética? Siete requisitos éticos. En: Pellegrini A, Macklin R, eds. Investigación en Sujetos Humanos: Experiencia Internacional. Santiago de Chile: Programa Regional de Bioética OPS/ OMS; 1999: 33-46.
} 
constó con claridad por parte del investigador en relación a las implicancias del estudio, que fueron expuestas detalladamente al participante, en forma previa, con el fin de escoger libremente sobre su participación en él. La investigación fue evaluada por el Comité de Ética e investigación del Hospital, y se utilizó Consentimiento Informado, en él se informó la finalidad, riesgos y beneficios.

\section{RESULTADOS}

Se presenta un cuadro de resultados, expresado en las categorías: contexto personal, contexto laboral, rol asistencial, educativo y administrativo. Los periodos se dividen para mayor objetividad del texto.

Cuadro 1. Historia del ejercicio profesional de enfermeras hospitalarias del sur de Chile (1940-1980).Contextos y Rol.

\begin{tabular}{|c|c|c|}
\hline Categorías & Años & Subcategorías \\
\hline \multirow[t]{2}{*}{ Contexto personal } & $1940-1960$ & $\begin{array}{l}\text { Valores y Formación: rigor-obediencia } \\
\text { Atención humanizada }\end{array}$ \\
\hline & $1970-1980$ & Compromiso y desarrollo profesional \\
\hline \multirow[t]{2}{*}{ Contexto laboral } & $1940-1960$ & Inicios arduos- carencia de enfermeras en el país \\
\hline & $1970-1980$ & $\begin{array}{l}\text { Sobrecarga laboral } \\
\text { Temor de expresar derechos laborales }\end{array}$ \\
\hline \multirow[t]{2}{*}{ Rol asistencial } & $1940-1960$ & $\begin{array}{l}\text { Cumplimiento de indicaciones médicas } \\
\text { Responsabilidad - calidad de atención } \\
\text { Actividades esencialmente técnicas, vacunación }\end{array}$ \\
\hline & $1970-1980$ & $\begin{array}{l}\text { Aplicación del proceso, delimitación de funciones } \\
\text { Realiza procedimientos especializados } \\
\text { Precariedad de recursos }\end{array}$ \\
\hline \multirow[t]{2}{*}{ Rol administrativo } & $1940-1960$ & $\begin{array}{l}\text { Organización de Servicios } \\
\text { Manejo de recursos humanos y materiales } \\
\text { Resolución de problemas, Supervisión }\end{array}$ \\
\hline & $1970-1980$ & $\begin{array}{l}\text { Influencia de reestructuración en salud } \\
\text { Liderazgo } \\
\text { Enfermería normativa }\end{array}$ \\
\hline Rol educativo & $\begin{array}{l}1940-1960 \\
1970-1980 \\
\end{array}$ & $\begin{array}{l}\text { Formación de auxiliares-Unidad de Capacitación } \\
\text { Perfeccionamiento profesional }\end{array}$ \\
\hline
\end{tabular}

La categoría contexto personal, período (1940-1960) en sus subcategorías, menciona valores y formación: rigor-obediencia y atención humanizada. Se insinúan fuertes influencias de los valores de la formación, la obediencia a las estructuras de poder, el sacrificio personal y la sumisión. El reconocimiento del otro es la principal característica del período, donde la identificación e influencia social están moduladas por la can- tidad de trabajo que la enfermera debía asumir. Así emergen los relatos: "Uno nace con ese cariño especial por la salud de los demás... amor por el prójimo..., porque uno quiere al enfermo. Para ingresar nos tomaban una prueba para ver si realmente nos gustaba...como era el carácter que teníamos para trabajar en salud. Si estábamos dispuestas a sacrificarnos". Otros relatos de rigor y obediencia: "Debido a que hacía frío y mala calefacción usábamos 
una capa ploma y el uniforme todo blanco completo con toca,... era estricto". Y en atención humanizada, [...] "el trato a los enfermos era preferencial, llegaba personal que no querían atender enfermos contagiosos o a los que estaban sucios, hubo que hacer una labor de conocimientos y actitudes".

En el segundo periodo (1970-1980) el contexto personal evoluciona hacia mayor conciencia del rol, expresado en un destacado compromiso profesional, liderazgo relacionado con eficientes relaciones interpersonales, que evolucionan desde un fenómeno de influencia en las personas, evidenciada a través de la comunicación, unión y capacidad de hacer cambios.

Contexto laboral: la etapa (1940-1960) es caracterizada por una situación de trabajo extremo y marcada carencia de enfermeras derivado de la insuficiencia de Escuelas formadoras en el país. Conjuntamente las condiciones laborales estaban fuertemente influenciadas por la realidad social y política de Chile. "No era fácil la tarea,... la iniciación fue dura". "No llegaban enfermeras... porque había escasez de ellas a nivel de todo Chile, cada año la distribución era de una o dos enfermeras... yo comencé con 6 enfermeras". "En $1967 . .$. por decisiones del Ministerio, no nos permitían elegir dónde trabajar... éramos designadas donde había mayor déficit". "Cuando recién empecé a ejercer la profesión teníamos un poco de temor porque en el Hospital habian tres enfermeras como máximo". "Para mí era un compromiso serio, no me sentía tan capacitada".

Esta etapa evoluciona hacia un período (1970-1980) de crisis, caracterizada por sobrecarga laboral, temor de expresar derechos laborales y marcada inestabilidad laboral relacionada con los acontecimientos históricos del período, Golpe y Gobierno Militar. En la última etapa se observa una conciencia de transición, reflejado en el reconocimiento del proceso de cambio, que parte con la identificación de los problemas (sociales, políticos y laborales) que rodean a la profesión y evo- luciona hacia un mayor compromiso con el ejercicio de la misma. Conjuntamente el período se caracteriza por presiones políticas, generando apremios al interior de la profesión, al imponer la extensión de las jornadas de trabajo y cierre de plantas estratégicas en los hospitales, lo que dificulta la capacidad de incorporar nuevas enfermeras. "Trabajábamos 6 días o 7 días..." "Los fines de semana veniamos voluntarias porque no se cancelaban horas extraordinarias... cubríamos de 8-14 horas para hacer las cosas más especiales". "Me fui a trabajar de 8-17 hrs, pero podía finalizar mi jornada a las 20 horas".

Este periodo se transformó en el de mayor inestabilidad laboral, y se manifiestan temores a la pérdida del trabajo, conflictos y amenazas; derivadas de las presiones políticas, “... existía miedo a perder el trabajo... en cualquier minuto te podían despedir" [...] "un día llegue atrasada y me mando a buscar el subdirector administrativo...y me dijo la próxima vez Ud. se va”.

Rol asistencial:, en los relatos, en la primera etapa, se alude a la enfermera como un profesional que frecuentemente era considerada como un trabajador mecánico, observándose déficit en el desarrollo profesional, conformidad, cooperación ciega y carencia de análisis, debido a la gran demanda de atención. En problemas de salud se menciona la alta tasa de mortalidad infantil por diarreas, problemas respiratorios y los accidentes en el hogar, específicamente las quemaduras en niños. "...veíamos morir niños con diarrea... la tasa de mortalidad de esa época... debe haber sido muy alta". "El 50 $\%$ de los pacientes eran quemados". Conjuntamente se menciona que los recursos eran muy limitados en cantidad y calidad, y de la precariedad de los mismos deriva el uso creativo de estrategias por parte de las enfermeras para optimizarlos. "Prácticamente a veces no había nada. Se trabajaba con jeringa de vidrio y agujas metálicas, que se lavaban y se les sacaba filo, los sueros venían en matraces de vidrio y los equipos de fleboclisis eran de 
goma". "Los lavábamos y los colocábamos en desinfectante". "Éramos enfermeras que trabajábamos con poco o nada y hacíamos maravillas con lo que teníamos". Simultáneamente, se indica la inquietud por el acatamiento de las indicaciones médicas y la realización de procedimientos: "Nos preocupábamos del cumplimiento de las indicaciones médicas". "Los procedimientos más delicados o especializados los realizábamos las enfermeras", "los de tipo inyectables". Especial relevancia es la realización de procedimientos derivados de la atención quirúrgica tales como arsenalería, suministrar anestesia, asistencia directa en cirugías y administración de quimioterapia; que eran responsabilidad exclusiva de las mismas: "...yo fui a hacer una especialización en anestesia". "Preparábamos las quimioterapias y las administrábamos sin guante". La tendencia en este ámbito fue tener mayor competencia técnica para la realización de procedimientos, destacando su responsabilidad y calidad de atención.

En la segunda etapa (1970-1980) se aprecia una mayor diferenciación de las actividades propias del rol y las delegadas en las auxiliares de Enfermería; es aquí donde el Proceso de Atención de Enfermería emerge como herramienta exclusiva de las enfermeras, explicitada a través de los Planes de Atención registrados en las Hojas de Enfermería: "Siempre la división del trabajo entre las enfermeras y las técnicos estaba bastante clara". "Se planificaba la atención y era una actividad propia de la enfermera y se realizaba en la Hoja de Enfermería”.

La situación de los recursos se modifica a raíz de la aparición de las Normas de Prevención de Infecciones Intrahospitalarias (IIH1980). "Apareció el SIDA 1982-83 fue ahí el gran vuelco, apareció material desechable y se eliminó la reesterilización por Norma Ministerial... se inició el Control de las Infecciones Intrahospitalarias y las primeras normas".

Rol administrativo: desde 1940-1960 el rol de las enfermeras estuvo caracterizado por la Habilitación y Organización de Ser- vicios, construyéndose posteriormente una Enfermería estructurada, donde el cargo de jefa de enfermeras del Hospital adquiere gran importancia; contradictoriamente las tareas administrativas fueron vistas como no propias de la enfermera, centradas en la solución de problemas de tipo domésticos y en el manejo de recursos humanos, principalmente orientando la supervisión como una estrategia de vigilancia directa de los trabajadores subalternos, verificando su actuar en su lugar de trabajo y no en la calidad de las atenciones brindadas por éstos. "...siempre había que organizar y habilitar Servicios". "Existía la Enfermera Jefa de Hospital, cargo de mucha importancia". "Siempre la enfermera ha manejado los insumos y recursos humanos". "La parte administrativa no era Enfermería propiamente tal". "Habia que ver si estaba trabajando el personal nuevo".

Durante las siguientes décadas (1970-80) acontece la Reestructuración en Salud, con énfasis en la Supervisión de Personal, relacionado con la aparición de las Normas de Prevención de Infecciones Intrahospitalarias. "Se administraban los Servicios de Enfermería con características de líder". "Supervisábamos al personal trabajando junto a ellos". Se insinúan las bases de los actuales Modelos de Gestión relacionados con la calidad y seguridad del paciente.

Rol educador: el inicio del período 1940'60 sentó precedente en la necesidad de formar personal de ayuda en las tareas de Enfermería, seleccionando personas sin instrucción previa. "Había gente que venía de la calle $y$ costó mucho formarla, debian tener conocimientos básicos y era complejo". En la década de 1950 se manifiesta mayor preocupación por instruir formalmente al personal auxiliar. "Después se dictaron Cursos de Auxiliares de Enfermería". Otro hito importante fue la llegada de las Internas de Enfermería, quienes realizaron actividades educativas y asistenciales y contribuyeron al fortalecimiento del rol docente. "Yo siempre tuve internas y les realizaba pasos prácticos". "Las alumnas obser- 
varon y adquirieron toda la práctica".

En la etapa siguiente se logra avanzar en la formación formal de auxiliares de Enfermería en la Unidad de Capacitación del Hospital, tal como lo menciona el relato siguiente: "Los Auxiliares de Enfermería se formaban en la Unidad de Capacitación y nosotras teníamos que capacitarlas".

Luego aparece mayor formación continua e información, emergiendo en el año 1965 la revista de Enfermería del Colegio de Enfermeras de Chile: "Nos preocupábamos, íbamos al menos a un Congreso al año". "Por el hecho de estar inscritas en el Colegio recibíamos la Revista... y recibiamos las Nursing".

Rol investigador: el estudio no arrojó hallazgos, debido al déficit de desarrollo y de la producción de conocimientos durante la época.

\section{DISCUSIÓN Y CONCLUSIÓN}

En los relatos descubrimos que la distribución de funciones y actividades derivadas del rol se modifican y sufren transiciones, de acuerdo a los contextos sociopolíticos, situación similar a lo explicitado en el Ejercicio Profesional de la Enfermeras, definido por el Colegio de Enfermeras de Chile como el conjunto de funciones cuyas actividades y tareas cambian continuamente (32).

De igual forma en los sujetos de estudio el ejercicio profesional, periodo 1940-1960, se caracteriza por valores sociales y alta vocación de servicio, características propias de las profesionales de la época que son coherentes con los requisitos de admisibilidad de las Escuelas de Enfermería de la época como: test de personalidad y aptitudes (33). Otra particularidad es la sumisión, obediencia y respeto hacia las estructuras de poder, manifestada en el estricto cumplimiento de normas y el recto uso del uniforme, que se explican por la hegemonía médica, donde en gran medida la enfermera es una ejecutante de órdenes médicas, y desarrolla su rol bajo su jerarquía (34). No obstante, los sujetos de estudio identifican cierto grado de autonomía en aspectos administrativos.

Otro aspecto que se relata en publicaciones, se refiere a la marcada preocupación por la limitada relación enfermera- paciente, debido al insuficiente número de profesionales que brindaban servicios en las instituciones públicas (50 a 100 pacientes por enfermera) (34), situación que se relaciona con los relatos, que enuncia la gran conciencia que existía en las ellas, por lo que asumen la responsabilidad de formar personal auxiliar de Enfermería, con énfasis en el trato humanizado y respeto a los pacientes.

En el rol asistencial, los textos se centran en la preocupación por el cumplimiento de las indicaciones médicas y la realización de procedimientos clínicos, hallazgos que concuerdan con lo planteado por Bocaz y Figueroa: "En Chile la Enfermería había evolucionado de oficio a profesión enmarcada en los contextos autoritarios y de obediencia ciega a las instituciones militares, religiosas y asistenciales" (35), y porque las profesionales mayoritariamente se desempeñan dentro de sistemas hospitalarios burocráticos, fluctuando entre fuerzas de poder médico y administrativo (36). En la segunda etapa (1970-1980) se describen diferencias en las actividades de las enfermeras y las delegadas a auxiliares de Enfermería, surgiendo el Proceso de Atención de Enfermería. La realización de procedimientos de arsenalería, suministrar anestesia, asistencia directa en cirugía y administración de quimioterapia eran de responsabilidad exclusiva de las mismas, tópico que coincide con la descripción de la función de la enfermera clínica de Pincheira, "ejecuta funciones que son del campo de los especialistas y que se han convertido en acciones de enfermería tales como: hemodiálisis, anestesia, manejo de máquinas de circulación extracorpórea, manejo de monitores, toma de electrocardiogramas (ECG) e interpretación y manejo de respiradores" (32). 
La situación de los recursos, que se describe como desproporcionada, entre los disponibles y las necesidades asistenciales del país (36), se ve afectada por las primeras políticas de extensión de la cobertura de los Servicios de Salud a las poblaciones rurales, y comienzan a emerger una serie de críticas a este tipo de atención. En un Informe sobre Salud del Banco Mundial (37) surge la afirmación: "Para aumentar la eficacia de los recursos y asegurar un acceso más equitativo a los cuidados de salud es necesario que los gobiernos reduzcan sus gastos en hospitales y personal altamente calificado y dediquen más recursos a dotar de personal los Servicios de Salud de menor nivel".

Conjuntamente, a partir del año 1980, a raíz de la aparición de las primeras Normas de Prevención de Infecciones Intrahospitalarias (IIH), y su implementación (38), que determinaba su ejecución inmediata y efecto manifiesto por medio de la Acreditación de Hospitales, supervisado por una Comisión Asesora a nivel Ministerial, constituida por el Decreto Supremo 023 del 6 de octubre de 1980 , se reconoce que las medidas que contribuyen a reducir la frecuencia y magnitud de las infecciones pueden demandar mayores gastos económicos, y que la mayoría se puede obtener respetando las recomendaciones de manejo de enfermos, distribución de salas, aislamiento y esterilización de instrumentos y equipo, entre otras. $\mathrm{Al}$ respecto, el Ministerio asistía con instrucciones para la implementación de Medidas de Prevención de Infecciones Intrahospitalarias desde 1977 (38), pero aparentemente la falta de recursos en los hospitales retrasaba su cumplimiento.

Posteriormente con la aparición de la epidemia de VIH/SIDA y específicamente con la notificación de seis personas, distribuidas en las regiones de Valparaíso, Biobío y Metropolitana (39), se desencadena una Alerta Sanitaria que favoreció la implementación de normas con estándares internacionales, en el manejo y manipulación de sangre y fluidos corporales, obteniéndose recursos materiales de mejor calidad y utilización de materiales desechables. Esta información es concordante con los relatos de las enfermeras respecto de que la escasez de recursos materiales e insumos y la aparición del SIDA fueron facilitadores en la adquisición de recursos.

En el rol administrativo, en la segunda etapa, debido al proceso político que vive el país, se reestructura el Sistema de Salud y se expresa con fuerza esta función del rol, en Enfermeras Clínicas y jefaturas, reconociendo al jefe como líder, con responsabilidad en la solución de problemas y supervisión de servicios, aspectos concordantes a lo señalado por el Colegio de Enfermeras, "el rol administrativo tiene entre sus funciones la planificación, dirección, control, supervisión, coordinación, evaluación de las actividades y prestaciones de la enfermera en las diferentes áreas de trabajo" (40).

En el rol educador, años 1940-60, manifestado por la necesidad de formar personal de ayuda (selección de personas sin instrucción previa), se reafirma que numerosas tareas de Enfermería eran realizadas por personas de limitada educación y que el público no se percataba que podría contar con un Servicio de Enfermería de mejor calidad (40). Debe señalarse que entre 1949-1959 los requisitos de admisión en las escuelas eran inferiores a la Educación Primaria, posteriormente se exige un aumento de la escolaridad exigida para el ingreso. En 1949 el 61\% de las escuelas exigía de nueve a 12 años de escolaridad; en 1959 el 91\% de ellas exigía ese requisito, los datos confirman que los Servicios de Enfermería demandaban un personal mejor preparado $(5,41)$.

Al año 1950 existían 56 egresadas de la Universidad de Chile, de la Escuela de Enfermería de los Servicios de beneficencia 23 y Escuela de Enfermería Carlos van Buren, 19 egresadas, con un total de 133 enfermeras para todo el país, por ello era necesario la designación de cargos desde el nivel central a lugares de mayor requerimiento.

En 1950- 1960, de acuerdo a lo manifesta- 
do, se logra instruir formalmente al personal auxiliar y colaboran internas de Enfermería, las cuales procedían de la Universidad Austral, ellas reciben influencias en el ejercicio profesional de la revista de Enfermería.

En el rol investigador no arrojó hallazgos, sin embargo el déficit de desarrollo y producción de conocimientos durante la época, según Figueroa, se relaciona al desinterés, falta de independencia y poder (36). Durante la década del 60 comienzan las primeras investigaciones realizadas por enfermeras y al mismo tiempo es el principal sujeto de estudio, lo que coincide con la naturaleza de las preocupaciones de la Enfermería de la época, donde el interés de la profesión estaba enfocado en la formación, las áreas del ejercicio profesional, las funciones que debía realizar y las situaciones que el ámbito laboral les imponía (42).

La práctica dominante era la práctica sanitaria, saneamiento y lucha contra las enfermedades transmisibles. El ejercicio profesional en el primer período nos habla de una práctica ligada al desarrollo de los hospitales (medicina curativa) y de dominio sanitario, se muestra la dureza de la profesión, sus delimitadas funciones y la vinculación al déficit de salubridad e higiene. La segunda etapa evoluciona en transiciones ocurridas al interior de la organización y del subsistema de la profesión Enfermería, recibiendo fuerte influencia del entorno sociopolítico del país.

El análisis global nos lleva a concluir que la práctica y el saber en el campo de salud están ligados a la transformación histórica del proceso de producción económica, siendo el factor determinante el tipo de práctica y educación en Enfermería.

\section{REFERENCIAS}

1. Mendes IAC, Ventura CAA, Trevizan MA. La Cooperación Técnica para el desarrollo de Liderazgos en Enfermería: La expe- riencia de una Escuela de Enfermería de Brasil. Rev. Panamericana de Enfermería [Internet] 2004 [citado 4 noviembre 2010]; 2(2): [155-59]. Disponible: http:// gepecopen.eerp.usp.br/files/artigos/Artigo336fin.pdf

2. Durán de Villalobos M. Aspectos conceptuales del cuidado de enfermería: Utilidad para la práctica y la investigación. II Congreso de Egresados. Universidad Nacional de Colombia; 2000.

3. Verderese O. Análisis de la Enfermería en la América Latina. Educ Méd Salud [Internet]. 1979 [citado 5 noviembre 2010]; 13(4): 315-38. Disponible en: http://hist. library.paho.org/Spanish/EMS/5145.pdf

4. Arouca da Silva AS. O Dilema Preventivista [Tesis Doctorado]. [São Paulo (Brasil)]: Faculdade de Ciencias Médicas, Universidade de Campinas; 1975.

5. Chagas AW. La educación de enfermeras en la América Latina. Bol Of Sanit Panam. 1952; 32(1): 48-57.

6. Molina C. Historia de la protección Social de Salud Chile. Santiago, Chile: Unidad Patrimonio Cultural. Ministerio de Salud; 2008.

7. Mardones RF. Salud Materno-Infantil últimos 30 años. Evolución de la atención médica. En: Medicina Infantil. Tomo I, Editorial Winter y Puentes, 1991.

8. Albala C, Vio F, Robledo A, Icaza G. La transición epidemiológica en Chile. Rev Med Chil. 1993; 121(12): 1446-55.

9. Mönckeberg F. Discurso en agradecimiento al Homenaje de la Sociedad Latinoamericana de Nutrición. Arch Latin Nutr. 1994; 44(5-S).

10. Mardones RF. Salud Materno-Infantil últimos 30 años. Evolución de la atención médica. En: Medicina Infantil. Tomo I, Editorial Winter y Puentes, 1991.

11. Illanes M. En el nombre del pueblo del Estado y la ciencia. Historia social de la salud pública en Chile 1880-1973. Santiago, Chile: Andros; 1993.

12. Centro Latinoamericano de Demografía 
(CELADE). Evolución de la mortalidad infantil en Chile: períodos 1940-1960 y 1961-1980. Santiago, Chile : Archivos CELADE. s.f.

13. Meneguello J, Rosselot E, Mardones RF. Rol de la Pediatría Clínica y Social en los Avances de Salud del Niño y la Familia, Chile 1900-1995. Pediatría al día. 1995; 11: 223-31.

14. Bocaz I, Figueroa M. Educación continuada y estudios de graduados. Rev. Enfermería Colegio de Enfermeras de Chile. 1980; 64: 3-7.

15. Rosselot E, Meneguello J. Salud de la familia y paternidad responsable, la experiencia de Chile, 1965-1988. Rev Med Chil.1990; 118: 330-38.

16. Figueroa M. Análisis crítico del ejercicio profesional. Rev. Enfermería Colegio de Enfermeras de Chile. 1976; 50: 4-9.

17. Monzón M. Adaptación de los Códigos Deontológicos de Enfermería nacionales de la UE al código ético y deontológico para la enfermería europea. Reduca [Internet] 2010 [citado 19 junio 2011]; 2(1): [7]. Disponible en: http://revistareduca. es/index.php/reduca-enfermeria/article/ view/126

18. Hernández A, Guardado C. La enfermería como disciplina profesional holística. Rev. Cubana Enfermer [Internet]. 2004 [citado 5 noviembre 2010]; 20 (2):5. Disponible en: http://bvs.sld.cu/revistas/enf/ vol20_2_04/enf07204.htm

19. Pincheira S. Bases para una política de enfermería. Rev. Enfermería Colegio de Enfermeras de Chile. 1978; 56: 29-32.

20. Gracia D. Desarrollo, aportación e imbricación de la enfermería en el avance tecnológico. Presentado en: I Jornada Nacional de Investigación en Historia de la enfermería: La enfermería en el siglo XX. de oficio a profesión, los momentos del cambio. Seminario Permanente en investigación en Historia de la Enfermería; 1992; Madrid.

21. Burns N, Grove K. Investigación en En- fermería. 3ra edición. Madrid, España: Elsevier; 2005. p. 338-466.

22. Martinelli ML. Seminario sobre metodologías qualitativas de pesquisa. Abertura. En $\mathrm{O}$ uso de abordagens qualitativas na pesquisa em Serviço Social. Um instigante desafio. São Paulo: Editora PUCSP, NEPI; 1994.

23. Moljo C. La Historia oral, como posibilidad de reconstrucción histórica, su relación con el Trabajo Social [Internet]. Centro Português de Investigação em História e Trabalho Social; sf [citado 5 diciembre 2011]. Disponible en: http:// www.cpihts.com/2003_07.31/hist_oral. htm

24. Grele R. Movimiento sin meta: problemas metodológicos y teóricos en la Historia Oral. En: Schwarzstein D. La Historia Oral, compilación. Buenos Aires (Argentina): CEAL; 2001.

25. Grele R. Envelopes of Sound. The Art of Oral History. New York: Greenwood Publishing Group; 1991. 283 p.

26. Benjamin W. Magia e Técnica, Arte e Política: Ensaios sobre literatura história da cultura. 7a ed. Vol. 1, Obras Escolhidas. São Paulo: Brasiliense; 1994.

27. Albert V. Manual de Historia Oral. 3ra ed. Rio de Janeiro: Editora FGV; 2005.

28. Hammer D, Wildavsky A. Entrevista semi-estructurada de final abierto. Aproximación a una guía operativa. En: Vilanova M, editor. Historia y Fuente Oral 4 Entrevistas... ¿para qué? Barcelona: Universitat de Barcelona Publicacions; 1990. p. 23-61.

29. Ullin P, Robinson E, Tolley E. Investigación aplicada en salud pública. Métodos cualitativos. Washington (D.C): OPS; 2006.

30. Delgado LAN. Historia oral. Memória, tempo, identidades. Belo Horizonte: autentica; 2006.

31. Guba E, Lincoln Y. Effective evaluation: improving the usefulness of evaluation results through responsive and natu- 
ralistic approaches. San Francisco: Jossey-Bass; 1981.

32. Pincheira S. Necesidades de Enfermería, Editorial. Rev. Enfermería Colegio de Enfermeras de Chile. 1977; 51: 3.

33. Lozier H. El problema de la selección de alumnas para las escuelas de Enfermería [Internet]. Washington (D.C.): Oficina Sanitaria Panamericana; 1951 [citado 5 diciembre 2011]. Disponible en: http:// hist.library.paho.org/Spanish/BOL/ v30n3p380.pdf

34. Hernández C. Estilos de liderazgo de enfermeras y enfermeros del Servicio Cántabro de Salud. Reduca [Internet]. 2010 [citado 2 noviembre 2010]; 2(1): 593606. Disponible en: http://www.revistareduca.es/index.php/reduca-enfermeria/ article/viewFile/197/219

35. Bocaz I, Figueroa M. Educación continuada y estudios de graduados. Rev. Enfermería Colegio de Enfermeras de Chile. 1980; 64: 3-7.

36. Figueroa M. Aspectos Dinámicos del trabajo en equipo. Rev. Enfermería Colegio de Enfermeras de Chile. 1977; 51: 4-15.

37. Banco Mundial. Salud. Documento de política sectorial. Washington (D.C.); 1975.
38. Ministerio de Salud Chile. Departamento de apoyo a los programas. Circular $\mathrm{N}^{\circ}$ 183. Control de Infecciones Intrahospitalarias. Santiago de Chile: Ministerio de Salud; 1980.

39. García M, Olea A. Evolución y situación epidemiológica de la infección por virus de inmunodeficiencia humana y síndrome de inmunodeficiencia adquirida en Chile. Rev Chilena Infectol [Internet]. 2008 [citado diciembre 2011]; 25(3): 162-70. Disponible en: http://www.scielo.cl/scielo.php?script $=$ sci_arttext\&pi$\mathrm{d}=$ S0716-10182008000300003

40. Colegio de Enfermeras. Rol de la enfermera enfermero. Rev. Enfermería Colegio de Enfermeras de Chile. 1976; 50: 38-40.

41. Organizacion Mundial de la Salud/Organización Panamericana de la Salud. Encuesta sobre las escuelas de Enfermería en América Latina, 1959. Publicación Científica No 62. Washington, D.C.: OPS; 1962.

42. Mendoza S, Klijn T. Organización y tendencias del conocimiento de enfermería en Chile. Rev Bras Enferm. 2004; 57(2): 143-51. 